J. Austral. Math. Soc. 25 (Series A) (1978), 45-65

\title{
GREEN'S RELATIONS FOR REGULAR ELEMENTS OF SANDWICH SEMIGROUPS, II; SEMIGROUPS OF CONTINUOUS FUNCTIONS
}

\author{
K. D. MAGILL, JR, and S. SUBBIAH \\ (Received 6 May 1975; revised 25 June 1977) \\ Communicated by T. E. Hall
}

\begin{abstract}
A sandwich semigroup of continuous functions consists of continuous functions with domains all in some space $X$ and ranges all in some space $Y$ with multiplication defined by $f g=f \circ \alpha \circ g$ where $\alpha$ is a fixed continuous function from a subspace of $Y$ into $X$. These semigroups include, as special cases, a number of semigroups previously studied by various people. In this paper, we characterize the regular elements of such semigroups and we completely determine Green's relations for the regular elements. We also determine the maximal subgroups and, finally, we apply some of these results to semigroups of Boolean ring homomorphisms.
\end{abstract}

Subject classification (Amer. Math. Soc. (MOS) 1970): primary 20M20, 54H15, 20M10; secondary 06A40.

\section{Introduction}

This paper is a continuation of Magill and Subbiah (1975) and we assume a certain familiarity with the notation, terminology and results of that paper. Let $X$ and $Y$ be topological spaces, let $\alpha$ be any continuous function with Dom $\alpha \subset Y$ and $\operatorname{Ran} \alpha \subset X$ and let $\mathscr{E}$ be any nonempty collection of subsets of $X$. Denote the collection of all continuous functions $f$ with $\operatorname{Dom} f \in \mathscr{E}$ and $\operatorname{Ran} f \subset Y$ by $S(X, Y, \alpha, \mathscr{E})$.

Definition (1.1) The family $\mathscr{E}$ is said to be $\alpha$-admissible if $f \circ \alpha \circ g \in S(X, Y, \alpha, \mathscr{E})$ whenever both $f, g \in S(X, Y, \alpha, \mathscr{E})$.

To say that $\mathscr{E}$ is $\alpha$-admissible is to say that $S(X, Y, \alpha, \mathscr{E})$ is a semigroup with the product $f g$ of two functions defined by $f g=f \circ \alpha \circ g$. When $\mathscr{E}$ is $\alpha$-admissible, $S(X, Y, \alpha, \mathscr{E})$ is, in the terminology of Magill and Subbiah (1975), a sandwich semigroup with sandwich function $\alpha$. We do not exclude the possibility of a topological space being empty in this paper but we assume throughout the paper that the spaces $X$ and $Y$ of any sandwich semigroup $S(X, Y, \alpha, \mathscr{E})$ are both nonempty. Such semigroups (for particular $\mathscr{E}$ ) have been discussed in Magill (1967).

This research was partially supported by the research foundation of the State University of New York. 
In Section 2, we discuss $\alpha$-admissible families and we obtain there various elementary results about such families. From Section 3 on, we assume that $\mathscr{E}$ is $\alpha$-admissible and hence that $S(X, Y, \alpha, \mathscr{E})$ is a semigroup. In Section 3 the regular elements of $S(X, Y, \alpha, \mathscr{E})$ are characterized and, in various instances, it is determined when the semigroups are regular. Green's relations for the regular elements of $S(X, Y, \alpha, \mathscr{E})$ are characterized in Section 4 and the maximal subgroups of $S(X, Y, \alpha, \mathscr{E})$ are determined in Section 5. In Section 6, sandwich semigroups of Boolean ring homomorphisms are treated. The main result characterizes, within a certain subclass, those semigroups which are regular. The proof relies on a result in Section 3 on sandwich semigroups of continuous functions.

\section{2. $\alpha$-Admissible families}

Throughout this section, $X$ and $Y$ are arbitrary topological spaces and $\alpha$ is any (nonempty) continuous function with $\operatorname{Dom} \alpha \subset Y$ and $\operatorname{Ran} \alpha \subset X$. As before, $\mathscr{E}$ is a nonempty collection of subsets of $X$ and $S(X, Y, \alpha, \mathscr{E})$ is the family of all continuous functions $f$ with $\operatorname{Dom} f \in \mathscr{E}$ and $\operatorname{Ran} f \subset Y$.

Proposition (2.1) The family $\mathscr{E}$ is $\alpha$-admissible if and only if $(\alpha \circ h)^{-1}(H) \in \mathscr{E}$ for each $H \in \mathscr{E}$ and $h \in S(X, Y, \alpha, \mathscr{E})$.

Proof. Suppose that $f, g \in S(X, Y, \alpha, \mathscr{E})$. Then $\operatorname{Dom} f \in \mathscr{E}$ and since

$$
\operatorname{Dom} f \circ \alpha \circ g=(\alpha \circ g)^{-1}(\operatorname{Dom} f) \text {, }
$$

it is immediate that $f \circ \alpha \circ g \in S(X, Y, \alpha, \mathscr{E})$.

On the other hand, suppose that $h$ belongs to $S(X, Y, \alpha, \mathscr{E})$ and $H \in \mathscr{E}$. Choose any point $p \in Y$ and define a function $f$ with $\operatorname{Dom} f=H$ by

$$
f(x)=p \text { for } x \in H \text {. }
$$

Then $f \in S(X, Y, \alpha, \mathscr{E})$ so that, by hypothesis, $f_{\circ} \alpha \circ h \in S(X, Y, \alpha, \mathscr{E})$. But

$$
\operatorname{Dom} f \circ \alpha \circ h=(\alpha \circ h)^{-1}(\operatorname{Dom} f)=(\alpha \circ h)^{-1}(H)
$$

and the proof is complete.

If one takes $\mathscr{E}$ to be the collection of all subsets of $X$, then $\mathscr{E}$ is $\alpha$-admissible regardless of the function $\alpha$ so that for each $\alpha$, there is always at least one $\alpha$-admissible family. The following result characterizes those $\alpha$ for which $\{X\}$ is $\alpha$-admissible.

Propositron (2.2) $\{X\}$ is $\alpha$-admissible if and only if $\operatorname{Dom} \alpha=Y$.

Proor. Suppose that $\{X\}$ is $\alpha$-admissible. We prove, by contradiction, that $\operatorname{Dom} \alpha=Y$. If $\operatorname{Dom} \alpha \neq Y$ then there is a point $p \in Y-\operatorname{Dom} \alpha$. Define $f$ by $f(x)=p$ for $x \in X$. Then $f^{2}=f_{\circ} \alpha \circ f=e$ (the empty function) which means Dom $f^{2}=\varnothing$. 
But then $\operatorname{Dom} f^{2} \notin\{X\}$ which is a contradiction so we conclude that $\operatorname{Dom} \alpha=Y$. It is immediate, from Proposition (2.1), that $\{X\}$ is $\alpha$-admissible whenever $\operatorname{Dom} \alpha=Y$.

The next result follows immediately from Proposition (2.1).

Proposition (2.3) If $\left\{\mathscr{E}_{\delta}\right\}_{\delta \in \Delta}$ is any collection of $\alpha$-admissible families and $\bigcap\left\{\mathscr{E}_{\delta}\right]_{\delta \in \Delta} \neq \varnothing$, then $\bigcap\left\{\mathscr{E}_{\delta}\right\}_{\delta \in \Delta}$ is also $\alpha$-admissible.

We denote the collection of all $\alpha$-admissible families by $\mathscr{A}(\alpha)$ and we partially order it by inclusion.

Propostrion (2.4) For any $\alpha, \mathscr{A}(\alpha)$ is a complete upper semilattice and a conditionally complete lower semilattice. It is a complete lattice if and only if $\operatorname{Dom} \alpha$ is a proper subset of $Y$.

Proof. It is immediate from Proposition (2.3) that $\mathscr{A}(\alpha)$ is a complete upper semilattice and a conditionally complete lower semilattice. Now it is known (Birkhoff, 1964, p. 49, Theorem 2), that a complete upper semilattice is a complete lattice if and only if it has a least element. We need only show that $\mathscr{A}(\alpha)$ has a least element if and only if Dom $\alpha$ is properly contained in $Y$. Suppose that Dom $\alpha$ is a proper subset of $Y$ and let $\mathscr{E}$ be $\alpha$-admissible. Choose any $H \in \mathscr{E}$ and $p \in Y-\operatorname{Dom} \alpha$ and define $f(x)=p$ for each $x \in H$. Then $f^{2}=f \circ \alpha \circ f=e$ (the empty function) so that $\varnothing=$ Dom $e \in \mathscr{E}$. Thus, when Dom $\alpha \neq Y$, every $\alpha$-admissible family contains the empty set and since $\{\varnothing\}$ is $\alpha$-admissible, it follows that $\{\varnothing\}$ is the least element of $\mathscr{A}(\alpha)$. On the other hand, if $\operatorname{Dom} \alpha=Y$, then $\{X\}$ is $\alpha$-admissible by Proposition (2.2). Since $\{\varnothing\}$ is also $\alpha$-admissible, we see that in this case, $\mathscr{A}(\alpha)$ can have no smallest element since no family of subsets of $X$ can be contained in both $\{X\}$ and $\{\varnothing\}$.

The next two results are easy consequences of Proposition (2.1).

Proposition (2.5) Suppose that Dom $\alpha$ is a closed subset of $Y$. Then the family $\mathscr{F}$ of all closed subsets of $X$ is $\alpha$-admissible.

Proposition (2.6) Suppose that Dom $\alpha$ is an open subset of $Y$. Then the family $\mathscr{G}$ of all open subsets of $X$ is $\alpha$-admissible.

We recall that a set is said to be clopen if it is both closed and open. The next result follows quickly from Proposition (2.1) or from Propositions (2.3), (2.5) and (2.6).

Proposition (2.7) Suppose that Dom $\alpha$ is a clopen subset of $Y$. Then the family $\mathscr{Z}$ of all clopen subsets of $X$ is $\alpha$-admissible. 
Some COMmEnts on NOTATION. At various times we want to deal with specific $\alpha$-admissible families and their corresponding sandwich semigroups. For any space $X$, the symbol $\mathscr{P}$ always denotes the family of all subsets of $X$, the symbol $\mathscr{F}$ always denotes the family of all closed subsets of $X$ and the symbol $\mathscr{Z}$ always denotes the family of all clopen subsets of $X$. The corresponding sandwich semigroups is denoted by $S(X, Y, \alpha, \mathscr{P}), S(X, Y, \alpha, \mathscr{F})$ and $S(X, Y, \alpha, \mathscr{Z})$. The first is a semigroup for any continuous $\alpha$ with $\operatorname{Dom} \alpha \subset Y$ and $\operatorname{Ran} \alpha \subset X$. Propositions (2.5) and (2.7) assure us that the second and third are semigroups if Dom $\alpha$ is respectively closed and clopen. When we discuss $S(X, Y, \alpha, \mathscr{F})$ we always assume that $\operatorname{Dom} \alpha$ is closed and when we discuss $S(X, Y, \alpha, \mathscr{Z})$ we assume that Dom $\alpha$ is clopen. When $Y=X$ and $\alpha$ is the identity map on $Y$, we use the simpler notation $S(X, \mathscr{P}), S(X, \mathscr{F})$ and $S(X, \mathscr{Z})$ in place of $S(X, Y, \alpha, \mathscr{P}), S(X, Y, \alpha, \mathscr{F})$ and $S(X, Y, \alpha, \mathscr{Z})$ respectively. Thus, $S(X, \mathscr{P})$ is the semigroup, under composition, of all continuous functions whose domains and ranges are both contained in $X$. $S(X, \mathscr{F})$ is the semigroup, under composition, of all continuous maps whose domain is a closed subset of $X$ and whose range is contained in $X$ and, similarly, $S(X, \mathscr{Z})$ is the semigroup, under composition of all continuous maps whose domain is a clopen subset of $X$ and whose range is contained in $X$. If $X$ is connected, then $S(X, \mathscr{Z})$ consists of all continuous functions mapping $X$ into $X$ together with the empty function.

We also want to consider the case where the $\alpha$-admissible class $\mathscr{E}$ consists solely of the space $X$ itself. Since we want $S(X, Y, \alpha, \mathscr{E})$ to be a semigroup, this forces Dom $\alpha$ to be all of $Y$ (Proposition (2.2)) and, in this case, $S(X, Y, \alpha, \mathscr{E})$ is just the sandwich semigroup of all continuous functions from $X$ into $Y$ (that is, the domains are all of $X)$. We denote this semigroup more simply by $S(X, Y, \alpha)$. When $X$ coincides with $Y$ and $\alpha$ is the identity map we have, of course, the semigroup $S(X)$ of all continuous selfmaps of $X$ under the binary operation of composition.

Finally, anytime we write $S(X, Y, \alpha, \mathscr{E})$ from now on, we assume that $\mathscr{E}$ is $\alpha$-admissible and that $X$ and $Y$ are nonempty.

\section{The regular elements of $S(X, Y, \alpha, \mathscr{E})$}

A retract of a topological space has the usual meaning here. That is, it is any subspace which is the range of a continuous retraction map of the space. We find it convenient to allow a topological space to be empty and to regard the empty function as a retraction map of the empty space. With this convention, a retract can then be empty. Of course, a retract of a nonempty space is itself nonempty. We also regard any function as mapping the empty set homeomorphically onto itself and we take its restriction to the empty set to be the empty function.

We conform to the terminology of Magill and Subbiah (1975) and for any sandwich semigroup $S(X, Y, \alpha, \mathscr{E})$ we define an $S$-retract of $Y$ to be any subspace of $Y$ which is the range of an idempotent element of the semigroup $S(X, Y, \alpha, \mathscr{E})$. 
In view of Lemma (3.1) of Magill and Subbiah (1975) every nonempty $S$-retract of $Y$ is a retract in the usual topological sense but the converse is far from true. The $S$-retracts are at least as dependent upon the behavior of the function $\alpha$ and the topological structure of $X$ as they are upon the topological structure of $Y$. They are characterized in the following

THEOREM (3.1) Let $S(X, Y, \alpha, \mathscr{E})$ be an arbitrary sandwich semigroup of continuous functions. Then a subspace of $Y$ is an S-retract if and only if $\alpha$ maps it homeomorphically onto a retract of some $H$ in $\mathscr{E}$.

Proof. Suppose first that $A \subset Y$ and that $\alpha$ maps $A$ homeomorphically onto $V$ which is a retract of some $H$ in $\mathscr{E}$. We first consider the case where $A=\varnothing$. Then $V=\varnothing$ and since the empty set is not a retract of any nonempty set, it follows that $H=\varnothing$. That is, $\varnothing$ belongs to the family $\mathscr{E}$. Thus, the empty function $e$ belongs to $S(X, Y, \alpha, \mathscr{E})$ and hence $A=\operatorname{Ran} e$ is an $S$-retract.

Now consider the case where $A \neq \varnothing$. Then $V \neq \varnothing$ and there exists a continuous retraction $v$ of $H$ whose range is $V$. Let $\alpha_{A}$ denote the restriction of $\alpha$ to $A$ and define $w=\alpha_{A}^{-1} \circ v$. Since $w$ is continuous and Dom $w \in \mathscr{E}$ it follows that $w \in S(X, Y, \alpha, \mathscr{E})$. Moreover, $\operatorname{Ran} w=A$ and $v$ is the identity on $V$ so that for any $y \in A$, we have

$$
(w \circ \alpha)(y)=\alpha_{A}^{-1}(v(\alpha(y)))=\alpha_{A}^{-1}(\alpha(y))=y .
$$

According to Lemma (3.1) of Magill and Subbiah (1975), this verifies that $A$ is an $S$-retract of $Y$.

Conversely, suppose that $A$ is an $S$-retract of $Y$. Then $\operatorname{Ran} v=A$ for some idempotent element $v$ of $S(X, Y, \alpha, \mathscr{E})$. If $A=\varnothing$ then, of course, $v=e$ which implies $\varnothing=$ Dom $e \in \mathscr{E}$. Since we have agreed to regard any function as mapping the empty set homeomorphically onto itself, it follows that $\alpha$ maps $A$ homeomorphically onto a retract of some set in $\mathscr{E}$.

In the case $A \neq \varnothing$, then $\operatorname{Dom} v=H \neq \varnothing$. It follows from Lemma (3.1) of Magill and Subbiah (1975) that $\alpha$ maps $A$ into $H$ so that $\alpha \circ v$ is a continuous selfmap of $H$. Moreover, for any $x \in \operatorname{Ran} \alpha \circ v$ we have $x=(\alpha \circ v)(a)$ for some $a \in H$ and we get

$$
(\alpha \circ v)(x)=(\alpha \circ v)((\alpha \circ v)(a))=(\alpha \circ v \circ \alpha \circ v)(a)=\left(\alpha \circ v^{2}\right)(a)=(\alpha \circ v)(a)=x .
$$

Hence $\alpha \circ v$ is a retraction and $\operatorname{Ran} \alpha \circ v$ is a retract of $H$. Now $v$ maps $\operatorname{Ran} \alpha \circ v$ into $A$ and $\alpha$ maps $A$ into $\operatorname{Ran} \alpha \circ v$. In addition, $\alpha \circ v$ is the identity on $\operatorname{Ran} \alpha \circ v$ and $v \circ \alpha$ is the identity on $A$ so we conclude that $\alpha$ maps $A$ homeomorphically onto $\operatorname{Ran} \alpha \circ v$.

In any Hausdorff space, retracts are closed. Of course there are usually many closed subsets which are not retracts. However, Corollary 2, page 281 of Kuratowski (1966) assures us that in nonempty 0-dimensional (that is, has a basis of clopen sets) 
metric spaces, the nonempty closed subsets coincide with the retracts so that from Theorem (3.1), we immediately get

CoRollary (3.2) Let $S(X, Y, \alpha, \mathscr{E})$ be a sandwich semigroup of continuous functions and suppose that $X$ is a 0-dimensional metric space. Then a nonempty subspace of $Y$ is an $S$-retract if and only if a maps it homeomorphically onto a closed subset of some $H$ in $\mathscr{E}$.

In the corollary, we cannot dispense with the requirement that the subspace of $Y$ be nonempty. The problem is that $\alpha$ will certainly map the empty set homeomorphically onto a closed subset of some $H$ in $\mathscr{E}$ for any sandwich semigroup $S(X, Y, \alpha, \mathscr{E})$. But the empty set can be an $S$-retract of $Y$ only if $e$ belongs to $S(X, Y, \alpha, \mathscr{E})$.

Now we need to know something of $S$-isomorphic subspaces of $Y$. Let $f$ be any element of $S(X, Y, \alpha, \mathscr{E})$ and suppose that $f_{\circ} \alpha$ maps $A$ into $B$. Recall (Magill and Subbiah, 1975, Definition (2.2)) that $f_{\circ} \alpha$ is said to map $A$ S-isomorphically onto $B$ if there exists a $g$ in $S(X, Y, \alpha, \mathscr{E})$ such that $g \circ \alpha$ maps $B$ into $A, g \circ \alpha \circ f \circ \alpha$ is the identity on $A$ and $f \circ \alpha \circ g \circ \alpha$ is the identity on $B$. The restriction of $f \circ \alpha$ to $A$ is referred to as an $S$-isomorphism from $A$ onto $B$ and $A$ and $B$ are said to be $S$-isomorphic. Although we did not specifically call attention to it in Magill and Subbiah (1975) we did not exclude the possibility of $A$ and $B$ being empty. The definition is such that $f_{\circ} \alpha$ will map the empty set $S$-isomorphically onto itself for any $f$ in $S(X, Y, \alpha, \mathscr{E})$. However, the restriction of each $f \circ \alpha$ to $\varnothing$ is the empty function $e$ so that there is only one $S$-isomorphism from $\varnothing$ onto $\varnothing$.

In view of the fact that we regard the empty function as mapping the empty set homeomorphically onto itself, it follows easily that $S$-isomorphic subspaces are homeomorphic but the converse is far from being true. For $S$-retracts, however, it is true. We gather these and some additional facts together in the next two theorems.

THEOREM (3.3) Let $S(X, Y, \alpha, \mathscr{E})$ be an arbitrary sandwich semigroup of continuous functions, let $A$ and $B$ be any two subsets of $Y$ and suppose that $f \circ \alpha$ maps $A$ $S$-isomorphically onto $B$. Then $\alpha$ maps $A$ homeomorphically onto $\alpha(A)$ and $B$ homeomorphically onto $\alpha(B)$ and $f \circ \alpha$ maps $A$ homeomorphically onto $B$.

Proof. Either $A$ and $B$ are both empty or they are both nonempty. In the former case the conclusion follows immediately and so suppose that they are nonempty. Since $f_{\circ} \alpha$ maps $A S$-isomorphically onto $B$, there exists a $g$ in $S(X, Y, \alpha, \mathscr{E})$ such that $g \circ \alpha$ maps $B$ into $A, g \circ \alpha \circ f_{\circ} \alpha$ is the identity on $A$ and $f \circ \alpha \circ g \circ \alpha$ is the identity on $B$. It is immediate that $f_{\circ} \alpha$ maps $A$ homeomorphically onto $B$. To see that $\alpha$ maps $A$ homeomorphically onto $\alpha(A)$, note that $g \circ \alpha \circ f$ maps $\alpha(A)$ into $A$. 
Since $\alpha \circ(g \circ \alpha \circ f)$ is the identity on $\alpha(A)$ and $(g \circ \alpha \circ f) \circ \alpha$ is the identity on $A$, it follows that $\alpha$ does indeed map $A$ homeomorphically onto $\alpha(A)$. The same sort of argument suffices to show that $\alpha$ also maps $B$ homeomorphically onto $\alpha(B)$.

THEOREM (3.4) Let $S(X, Y, \alpha, \mathscr{E})$ be an arbitrary sandwich semigroup of continuous functions, let $A$ be any subset of $Y$ and let $B$ be an $S$-retract of $Y$. Then $f_{\circ} \alpha$ maps $A$ S-isomorphically onto $B$ if and only if it maps $A$ homeomorphically onto $B$. Furthermore, two $S$-retracts of $Y$ are S-isomorphic if and only if they are homeomorphic.

Proof. Let $B$ be an $S$-retract of $Y$. It follows from the previous theorem that if $f \circ \alpha$ maps $A S$-isomorphically onto $B$, then it maps $A$ homeomorphically onto $B$. Conversely, suppose that $f \circ \alpha$ maps $A$ homeomorphically onto $B$ and denote the restriction of $f \circ \alpha$ to $A$ by $t$. Since $B$ is an $S$-retract, there is an idempotent element $w$ in $S(X, Y, \alpha, \mathscr{E})$ such that $\operatorname{Ran} w=B$. Then $t^{-1}$ o $w$ belongs to $S(X, Y, \alpha, \mathscr{E})$ and since $w_{\circ} \alpha$ is the identity on $B$ (Magill and Subbiah, 1975, Lemma (3.1)) it readily follows that $\left(t^{-1} \circ w\right) \circ \alpha$ maps $B$ into $A,\left(t^{-1} \circ w\right) \circ \alpha \circ f \circ \alpha$ is the identity on $A$ and $f \circ \alpha \circ\left(t^{-1} \circ w\right) \circ \alpha$ is the identity on $B$. Consequently, $f \circ \alpha$ maps $A S$-isomorphically onto $B$.

Now suppose that both $A$ and $B$ are $S$-retracts of $Y$. Theorem (3.3) assures us that if they are $S$-isomorphic, then they must be homeomorphic. Suppose, conversely, that they are homeomorphic and let $t$ be any homeomorphism from $A$ onto $B$. Since they are both $S$-retracts, there exist idempotents $v$ and $w$ in $S(X, Y, \alpha, \mathscr{E})$ such that $\operatorname{Ran} v=A$ and $\operatorname{Ran} w=B$. Then $t \circ v$ and $t^{-1} \circ w$ both belong to $S(X, Y, \alpha, \mathscr{E}), t \circ v$ maps $A$ into $B$ and $t^{-1} \circ w$ maps $B$ into $A$. Moreover, $v \circ \alpha$ is the identity on $A$ and $w \circ \alpha$ is the identity on $B$ and so it readily follows that $\left(t^{-1} \circ w\right) \circ \alpha \circ(t \circ v) \circ \alpha$ is the identity on $A$ and $(t \circ v) \circ \alpha \circ\left(t^{-1} \circ w\right) \circ \alpha$ is the identity on $B$. Hence, $A$ and $B$ are $S$-isomorphic.

Now we are in a position to characterize the regular elements of $S(X, Y, \alpha, \mathscr{E})$.

THEOREM (3.5) The following statements about an element $f$ in the sandwich semigroup $S(X, Y, \alpha, \mathscr{E})$ are equivalent.

(3.5.1) $f$ is regular.

(3.5.2) $\operatorname{Ran} f$ is an S-retract of $Y$ and there exists an S-retract $A$ of $Y$ such that $f \circ \alpha$ maps $A$ homeomorphically onto $\operatorname{Ran} f$.

(3.5.3) Ran $f$ is an $S$-retract of $Y$ and $f_{\circ} \alpha$ maps some subspace of $Y$ homeomorphically onto $\operatorname{Ran} f$.

Proof. This follows from Theorem (3.2) of Magill and Subbiah (1975) and Theorem (3.4) of this paper.

The next result shows that a large number of sandwich semigroups of continuous functions fail to be regular. 
THEOREM (3.6) Let $X$ be a completely regular Hausdorff space and suppose that $Y$ contains an arc $A$ such that $\alpha(A) \subset H$ for some $H \in \mathscr{E}$ which has more than one point. Then $S(X, Y, \alpha, \mathscr{E})$ is not regular.

Proof. First consider the case where $\alpha(A)$ consists of a single point. Since $H$ is completely regular and Hausdorff, there exists a continuous function $f$ mapping $H$ into $A$ such that $\operatorname{Ran} f$ contains more than one point. Then $f$ belongs to $S(X, Y, \alpha, \mathscr{E})$ but according to Theorem (3.1) Ran $f$ cannot possibly be an $S$-retract of $Y$ so that $f$ is not regular by Theorem (3.5).

Now we consider the case where $\alpha(A)$ consists of more than one point. Since $H$ is completely regular and Hausdorff and $\alpha(A)$ is connected, there exists a continuous map $g$ from $H$ onto the closed unit interval $I$. Let $f$ be any continuous map from $I$ onto $I$ which is not injective on any nondegenerate subinterval. For example, any continuous nowhere differentiable function which maps $I$ onto $I$ has this property. Finally, let $h$ be any continuous map from $I$ onto $A$. Then $h \circ f \circ g$ belongs to $S(X, Y, \alpha, \mathscr{E})$ but it is not regular since $h \circ f \circ g \circ \alpha$ does not map any subspace of $Y$ homeomorphically onto $\operatorname{Ran} h \circ f \circ g$ and this, of course, violates condition (3.5.3). To see that $h \circ f \circ g \circ \alpha$ maps no subspace of $Y$ homeomorphically onto $\operatorname{Ran} h \circ f \circ g$, note first that $\operatorname{Ran} h \circ f \circ g=A$ which is an $\operatorname{arc}$ and let $B$ be any $\operatorname{arc}$ in $Y$. If $g(\alpha(B))$ is a point, then so is $h \circ f \circ g \circ \alpha(B)$ and hence $h \circ f \circ g \circ \alpha$ does not map $B$ homeomorphically onto $A$. If $g(\alpha(B))$ is not a point, it is a nondegenerate subinterval of $I$ since it is connected. But then $f$ is not injective on $g(\alpha(B))$ so that $h \circ f \circ g \circ \alpha$ is not injective on $B$ and in this case as well, $h \circ f \circ g \circ \alpha$ does not map $B$ homeomorphically onto $A$.

CoRollary (3.7) Let $X$ be completely regular and Hausdorff and suppose that $Y$ contains an arc. Then $S(X, Y, \alpha)$ is regular if and only if $X$ consists of a single point.

Proof. If $X$ consists of a single point, then $S(X, Y, \alpha)$ is a left zero semigroup and is certainly regular. On the other hand, if $X$ does not consist of a single point, it follows immediately from Theorem (3.6) that $S(X, Y, \alpha)$ is not regular.

The next result shows, among other things, that there do exist regular sandwich semigroups $S(X, Y, \alpha)$ with nontrivial domain spaces $X$.

THEOREM (3.8) Let $X$ be a compact 0-dimensional Hausdorff space and let $Y$ be discrete. Then $S(X, Y, \alpha)$ is a regular semigroup if and only if one of the following conditions is satisfied.

(3.8.1) $X$ consists of one point,

(3.8.2) $Y$ consists of one point,

(3.8.3) $\alpha$ is injective and $\operatorname{Ran} \alpha$ is a dense subset of $X$. 
Proof. If (3.8.1) holds, then $S(X, Y, \alpha)$ is a left zero semigroup and hence is regular. If (3.8.2) holds then the semigroup consists of one constant function and is certainly regular. Now suppose that (3.8.3) holds and let $f$ be any element of $S(X, Y, \alpha)$. Since $X$ is compact and $Y$ is discrete, $\operatorname{Ran} f$ is finite and we denote the points in it by $\left\{y_{i}\right\}_{i=1}^{N}$. Then $f^{-1}\left(y_{i}\right)$ is a nonempty clopen subset of $X$ which intersects $\operatorname{Ran} \alpha$ since the latter is dense. Choose any $z_{i} \in Y$ such that $\alpha\left(z_{i}\right) \in f^{-1}\left(y_{i}\right)$. According to Theorem (3.1), both $\left\{z_{i}\right\}_{i=1}^{N}$ and $\left\{y_{i}\right\}_{i=1}^{N}$ are $S$-retracts and it follows from Theorem (3.4) that $f_{\circ} \alpha$ maps the former $S$-isomorphically onto the latter. Hence $f$, and $S(X, Y, \alpha)$ as well, are regular by Theorem (3.5).

Now suppose that $S(X, Y, \alpha)$ is regular. We suppose that neither (3.8.1) nor (3.8.2) holds and we show by contradiction that (3.8.3) must then hold. Assume that $\alpha(p)=\alpha(q)$ for two distinct points $p$ and $q$ of $Y$. Since $X$ is 0-dimensional and has more than one point, there exists an $f$ in $S(X, Y, \alpha)$ such that $\operatorname{Ran} f=\{p, q\}$. It is immediate from Theorem (3.1) that $\operatorname{Ran} f$ is not an $S$-retract and this contradicts Theorem (3.5). Thus, $\alpha$ must be injective. Now assume that $\operatorname{Ran} \alpha$ is not dense in $X$. Then there exist two nonempty disjoint clopen subsets $A$ and $B$ of $X$ such that $A \cup B=X$ and $\operatorname{Ran} \alpha \subset A$. Since $Y$ has more than one point, we may choose two distinct points $a$ and $b$ in $Y$ and define $g(x)=a$ for $x \in A$ and $g(x)=b$ for $x \in B$. Then $g \circ \alpha$ cannot possibly map a subset of $Y S$-isomorphically onto Ran $g$ since, in fact, Ran $g \circ \alpha=\{a\}$. Again Theorem (3.5) is contradicted and we conclude that $\operatorname{Ran} \alpha$ is dense in $X$.

EXAMPLE. Let $Y$ be discrete, let $\beta Y$ denote the Stone-Cech compactification of $Y$ and let $T(\beta Y, Y)$ denote the semigroup, under composition, of all continuous functions which map $\beta Y$ into its subspace $Y$. The semigroup $T(\beta Y, Y)$ is regular. This follows immediately from Theorem (3.8) by taking $X=\beta Y$ and $\alpha$ to be the identity map on $Y$.

Now we look at the semigroups $S(X), S(X, \mathscr{F}), S(X, \mathscr{Z})$ and $S(X, \mathscr{P})$ in more detail. From Theorem (3.6) we immediately get the following

COROLLARY (3.9) Suppose that $X$ is a completely regular Hausdorff space which contains an arc. Then none of the semigroups $S(X), S(X, \mathscr{F}), S(X, \mathscr{Z})$ are regular.

We could have included $S(X, \mathscr{P})$ in the statement of the previous corollary but we did not since we can say considerably more about it.

THEOREM (3.10) Suppose that $X$ is Hausdorff. Then $S(X, \mathscr{P})$ is regular if and only if $X$ is discrete.

Proof. First of all, if $X$ is discrete, then $S(X, \mathscr{P})$ is simply the semigroup, under composition, of partial functions on $X$ and it is known that this semigroup is regular. At any rate, this follows immediately from Theorem (3.5) of this paper. 
Now suppose that $X$ is not discrete. Since $X$ is Hausdorff one can choose a nondiscrete subset $A$ of $X$ and a point $p$ in $X$ such that $p \notin \operatorname{cl}_{X} A$. Let $q$ be any limit point of $A$ contained in $A$ and let

$$
B=[A-\{q\}] \cup\{p\} .
$$

Define a bijection $f$ from $B$ onto $A$ by $f(p)=q$ and $f(x)=x$ for $x \in B-\{p\}$. Then $f$ is continuous but it is certainly not a homeomorphism since $q$ is a limit point of $A$ but $p$ is not a limit point of $B$. The result now follows from Theorem (3.5).

Corollary (3.9) certainly indicates that $S(X), S(X, \mathscr{F})$ and $S(X, \mathscr{Z})$ are not often regular. Although we cannot hope to get a result for these semigroups like the previous result on $S(X, \mathscr{P})$, the next theorem still lends support to the fact that $S(X), S(X, \mathscr{F})$ and $S(X, \mathscr{Z})$ are seldom regular.

THEOREM (3.11) The following statements about a 0-dimensional metric space $X$ are equivalent.

(3.11.1) $S(X)$ is regular;

(3.11.2) $S(X, \mathscr{F})$ is regular;

(3.11.3) $S(X, \mathscr{Z})$ is regular;

(3.11.4) $X$ is either discrete or is the one-point compactification of the countably infinite discrete space.

Proof. We first show that (3.11.4) implies (3.11.2). Suppose that $X$ is discrete. Then $S(X, \mathscr{F})$ is the semigroup of all partial functions on $X$ and as we have already noted, this semigroup is regular. Now suppose that $X$ is the one-point compactification of the countably infinite discrete space and let $f$ be any function in $S(X, \mathscr{F})$. Since $\operatorname{Dom} f$ is closed, it is compact and hence $\operatorname{Ran} f$ is compact. If $\operatorname{Ran} f$ is finite, simply choose a point from each set of the decomposition of $\operatorname{Dom} f$ induced by $f$ and denote the resulting finite set by $A$. Then $f$ maps $A$ homeomorphically onto Ran $f$ and it follows from Theorem (3.5) that in this case, $f$ is regular. Just a bit more care must be taken if $\operatorname{Ran} f$ is infinite. When this is the case, the unique limit point $\infty$ must belong to $\operatorname{Ran} f$ since it is compact. For each $y \in \operatorname{Ran} f-\{\infty\}$, choose any $x_{y} \in f^{-1}(y)$ and let $A=\left\{x_{y}: y \in \operatorname{Ran} f-\{\infty\}\right\} \cup\{\infty\}$. Then $f$ maps $A$ homeomorphically onto $\operatorname{Ran} f$ and in this case too, it follows from Theorem (3.5) that $f$ is regular. Thus, $S(X, \mathscr{F})$ is a regular semigroup.

Now we show that (3.11.2) implies (3.11.1). Let $f$ be any element in $S(X)$. Since the latter is a subsemigroup of $S(X, \mathscr{F})$ which is assumed to be regular, there exists a $g$ in $S(X, \mathscr{F})$ such that $f \circ g \circ f=f$. Possibly $g$ may not belong to $S(X)$ which simply means that Dom $g \neq X$ but since Dom $g$ is closed one can, in view of Corollary 3, p. 281, of Kuratowski (1966), continuously extend $g$ to a function $\hat{g}$ in $S(X)$. Then $f \circ \hat{g} \circ f=f$ and we conclude that $S(X)$ is regular. 
Now we show that (3.11.1) implies (3.11.4). We assume that $X$ is not discrete and we show that this forces it to be the one-point compactification of the countably infinite discrete space. First of all, Corollary (3.5) of Magill and Subbiah (1974) assures us that the semigroup of all continuous selfmaps of a noncompact 0-dimensional metric space is regular if and only if the space is discrete. So this, together with the assumption that $X$ is not discrete implies that $X$ is compact. We show, by contradiction, that $X$ has only one limit point. Since it is compact and not discrete it must have at least one. Suppose it has more. We choose any two and denote them by $p$ and $q$. Since $X$ is first countable, there exists sequences $\left\{x_{n}\right\}_{n=1}^{\infty}$ and $\left\{y_{n}\right\}_{n=1}^{\infty}$ converging to $p$ and $q$ respectively. There is no loss in generality in assuming that all the points involved are distinct. Let

$$
A=\{p, q\} \cup\left\{x_{n}\right\}_{n=1}^{\infty} \cup\left\{y_{n}\right\}_{n=1}^{\infty}
$$

and choose mutually disjoint clopen sets $\left\{G_{n}\right\}_{n=1}^{\infty o}$ and $\left\{H_{n}\right\}_{n=1}^{\infty}$ so that $G_{n} \cap A=\left\{x_{n}\right\}$, $H_{n} \cap A=\left\{y_{n}\right\}, \lim \operatorname{Diam} G_{n}=0$ and $\lim \operatorname{Diam} H_{n}=0$ where Diam means diameter. Now define a selfmap $f$ of $X$ by

$$
\begin{array}{ll}
f(x)=y_{2 n-1} & \text { for } x \in H_{n}, \\
f(x)=y_{2 n} & \text { for } x \in G_{n}, \\
f(x)=q & \text { for } x \in X-\cup\left[G_{n} \cup H_{n}\right]_{n=1}^{\infty} .
\end{array}
$$

Since all the $G_{n}$ and $H_{n}$ are clopen, it is immediate that $f$ is continuous at any point in $G_{n}$ or $H_{n}$. To see that $f$ is continuous at $q$, let $V$ be any open set containing $q$. Then there exists a positive integer $N$ such that $y_{n} \in V$ for $n \geqslant N$ and one easily verifies that

$$
V-\left[\bigcup\left\{G_{i} \cup H_{i}\right\}_{i=1}^{N}\right.
$$

is a neighborhood of $q$ which $f$ carries into $V$. The same sort of argument serves to verify that $f$ is also continuous at the point $p$. Finally, let us consider a point $z$ different from both $p$ and $q$ and not belonging to any $G_{n}$ or $H_{n}$. First choose a clopen neighborhood $W$ of $z$ which excludes both $p$ and $q$. Then $W$ can intersect only finitely many of the sets $\left\{G_{n}\right\}_{n=1}^{\infty}$. Assume the contrary. Then we can choose $w_{n_{j}} \in G_{n_{j}} \cap W$ for infinitely many $n_{j}$. But $\lim x_{n_{j}}=p$ and $\lim \operatorname{Diam} G_{n_{j}}=0$ together imply that $p$ is a limit point of $W$ which is a contradiction since $W$ is clopen and does not contain $p$. In a similar manner, $W$ can intersect only finitely many of the sets $\left\{H_{n}\right\}_{n=1}^{\infty}$. Hence, there is a positive integer $N$ such that $W \bigcap\left[G_{n} \cup H_{n}\right]=\varnothing$ for $n>N$ and it follows that

$$
W-\left[G_{i} \cup H_{i}\right]_{i=1}^{N}
$$

is a clopen subset of $X$ containing $z$ which $f$ maps entirely into the point $q$. Consequently, $f$ is continuous at $z$ and hence on all $X$. That is, $f \in S(X)$.

Our next step is to show that $f$ does not map any subspace of $X$ homeomorphically onto $\operatorname{Ran} f$. Suppose that $f$ maps $B$ bijectively onto $\operatorname{Ran} f$. Then $B \cap G_{n}$ consists of exactly one point which we denote by $a_{n}$ and $B \cap H_{n}$ consists of exactly one point 
which we denote by $b_{n}$. Now $B \cap f^{-1}(q)$ also consists of exactly one point and we denote it by $t$. Thus,

$$
B=\{t\} \cup\left\{a_{n}\right\}_{n=1}^{\infty} \cup\left\{b_{n}\right\}_{n=1}^{\infty} .
$$

But $\lim x_{n}=p$ and $\lim \operatorname{Diam} G_{n}=0$ together imply that $\lim a_{n}=p$. For similar reasons, $\lim b_{n}=q$. Hence both $p$ and $q$ are limit points of $B$. However, each $a_{n}$ and each $b_{n}$ is isolated in $B$ so that the only possibility for a limit point is the point $t$. It is now apparent that $B$ cannot contain both $p$ and $q$ and hence is not compact. Consequently, $f$ does not map any subspace of $X$ homeomorphically onto Ran $f$ and according to Theorem (3.5) cannot be regular. This is the contradiction we seek and so we may conclude that $X$ has exactly one limit point which we denote by $\infty$. Since $X$ is compact it is immediate that it is the one-point compactification of a discrete space. However, $X$ is also metrizable so that according to Theorem (8.6), p. 247 of Dugundji (1966), $X-\{\infty\}$ is second countable. Since $X-\{\infty\}$ is also discrete, all this forces it to be countably infinite.

Now, at this point, we have shown that (3.11.1), (3.11.2) and (3.11.4) are all equivalent. One can verify that (3.11.4) implies (3.11.3) in exactly the same way that we verify that (3.11.4) implies (3.11.2). Similarly, one verifies that (3.11.3) implies (3.11.1) in the same manner that we showed that (3.11.2) implies (3.11.1). In fact, it is even a bit easier to show that (3.11.3) implies (3.11.1) in the sense that in this case, Dom $g$ will be clopen so that $g$ can be extended to $\hat{g}$ in $S(X)$ without appealing to Corollary 3, p. 281 of Kuratowski (1966). With these observations, we conclude the proof.

\section{Green's relations for regular elements}

In order to verify the results in this section we essentially have only to piece together various results from Magill and Subbiah (1975) and the previous section of this paper. We formally state all the results and then we discuss their proofs. We recall that for any function $f$, the symbol $\pi(f)$ denotes its decomposition. That is, $\pi(f)=\left\{f^{-1}(y): y \in \operatorname{Ran} f\right\}$.

THEOREM (4.1) Let $f$ and $g$ be regular elements of $S(X, Y, \alpha, \mathscr{E})$ and suppose, in addition, that $\operatorname{Dom} f=\operatorname{Dom} g$. Then $f$ belongs to the left ideal generated by $g$ if and only if $\pi(g)$ refines $\pi(f)$. Any two regular elements $h$ and $k$ of $S(X, Y, \alpha, \mathscr{E})$ are $\mathscr{L}$-related if and only if $\pi(h)=\pi(k)$.

THEOREM (4.2) Let $f$ and $g$ be any two regular elements of $S(X, Y, \alpha, \mathscr{E})$. Then $f$ belongs to the right ideal generated by $g$ if and only if $\operatorname{Ran} f \subset \operatorname{Ran} g$. Consequently, $f$ and $g$ are $\mathscr{R}$-related if and only if $\operatorname{Ran} f=\operatorname{Ran} g$.

TheOREM (4.3) Two regular elements $f$ and $g$ of $S(X, Y, \alpha, \mathscr{E})$ are $\mathscr{D}$-related if and only if $\operatorname{Ran} f$ is homeomorphic to $\operatorname{Ran} g$. 
TheOREM (4.4) Let $f$ and $g$ be any two regular elements of $S(X, Y, \alpha, \mathscr{E})$. Then $f$ belongs to the two-sided ideal generated by $g$ if and only if $\operatorname{Ran} f$ is homeomorphic to an $S$-retract of $Y$ which is contained in Rang. Consequently, $f$ and $g$ are $\mathscr{J}$-related if and only if the range of each contains an S-retract which is homeomorphic to the range of the other.

We recall that if one element in a $\mathscr{D}$-class is regular then they are all regular and such classes are called regular $\mathscr{D}$-classes. The next result tells us how we can get all of the regular $\mathscr{D}$-classes of $S(X, Y, \alpha, \mathscr{E})$.

THEOREM (4.5) Let $A$ be any subspace of $Y$ with the property that $\alpha$ maps it homeomorphically onto a retract of some $H$ in $\mathscr{E}$. Let $D_{A}$ consist of all those functions $f$ in $S(X, Y, \alpha, \mathscr{E})$ such that $\alpha$ maps $\operatorname{Ran} f$ homeomorphically onto a retract of some $H$ in $\mathscr{E}$ and $f \circ \alpha$ maps some subset of $Y$ homeomorphically onto $\operatorname{Ran} f$. Then $D_{A}$ is a regular $\mathscr{D}$-class of $S(X, Y, \alpha, \mathscr{E})$ and all regular $\mathscr{D}$-classes of $S(X, Y, \alpha, \mathscr{E})$ are obtained in exactly this manner.

VERIFICATION OF THEOREMS (4.1)-(4.5). Theorem (4.1) follows immediately from Theorems (3.6) and (3.7) of Magill and Subbiah (1975) and Theorem (4.2) follows from Theorem (3.9) of that paper. Theorem (4.3) follows from Theorem (3.10) of that paper and Theorems (3.4) and (3.5) of this paper. Theorem (4.4) follows from Theorem (3.12) of that paper and Theorem (3.3) of this paper. Finally, Theorem (4.5) follows from Theorem (3.11) of that paper and Theorems (3.1), (3.3) and (3.5) of this paper. We elaborate a bit more on the latter. Theorem (3.11) of Magill and Subbiah (1975) tells us that in order to get a regular $\mathscr{D}$-class of $S(X, Y, \alpha, \mathscr{E})$ we first choose any $S$-retract of $Y$ and according to Theorem (3.1), this is simply any subspace of $Y$ which $\alpha$ maps homeomorphically onto a retract of some $H$ in $\mathscr{E}$. Then we take $D_{A}$ to be all those functions $f$ such that $\operatorname{Ran} f$ is $S$-isomorphic to $A$ and $f \circ \alpha$ maps some subset of $Y S$-isomorphically onto $\operatorname{Ran} f$. Such $D_{A}$ are, according to Theorem (3.11) of Magill and Subbiah (1975) precisely the regular $\mathscr{D}$-classes of $S(X, Y, \alpha, \mathscr{E})$. Now the requirement that $f \circ \alpha$ map some subset of $Y S$-isomorphically onto $\operatorname{Ran} f$ is just condition (3.2.3) of Theorem (3.2) of the latter paper and although it is not formally stated, it follows immediately from the fact that (3.2.2) and (3.2.3) are equivalent that (3.2.3) is also equivalent to the requirement that $\operatorname{Ran} f$ be an $S$-retract of $Y$ and that $f_{\circ} \alpha$ map some subset of $Y S$-isomorphically onto Ranf. But according to Theorems (3.1) and (3.4) of this paper, this is equivalent to requiring that $\alpha \operatorname{map} \operatorname{Ran} f$ homeomorphically onto a retract of some $H$ in $\mathscr{E}$ and $f \circ \alpha$ map some subset of $Y$ homeomorphically onto $\operatorname{Ran} f$. This verifies Theorem (4.5).

Now we interpret what the previous results mean for the semigroups $S(X)$, $S(X, \mathscr{F}), S(X, \mathscr{P})$ and $S(X, \mathscr{Z})$. The statements concerning the $\mathscr{L}, \mathscr{R}$ and 
$\mathscr{D}$-relations are identical for the four semigroups and are simply the statements of Theorems (4.1)-(4.3). However, the statements for the $\mathscr{J}$-relation differ for the four semigroups and the reason for this is that the statement concerning the $\mathscr{J}$-relation explicitly involves a reference to $S$-retracts and these differ depending upon the semigroup. For $S(X)$, an $S$-retract is just a retract of $X$ in the usual topological sense and so we get

COROLlary (4.6) Let $X$ be any topological space and let $f$ and $g$ be any two regular elements of $S(X)$. Then $f$ belongs to the two-sided ideal generated by $g$ if and only if $\operatorname{Ran} f$ is homeomorphic to a retract which is contained in Rang. The two functions are $\mathscr{J}$-equivalent if and only if the range of each contains a retract which is homeomorphic to the range of the other.

Since the retracts of 0 -dimensional metric spaces are precisely the nonempty closed subsets of the space (Kuratowski, 1966, p. 281) we also get:

COROLlary (4.7) Let $X$ be a 0-dimensional metric space and let $f$ and $g$ be regular elements of $S(X)$. Then $f$ belongs to the left ideal generated by $g$ if and only if Rang contains a closed subspace which is homeomorphic to $\operatorname{Ran} f$. The two functions are $\mathscr{J}$-equivalent if and only if the range of each contains a closed subspace which is homeomorpuic to the range of the other.

Now we consider the case for the semigroup $S(X, \mathscr{F})$. The verification of the next lemma is easy and we omit it.

LEMma (4.8) For a Hausdorff space $X$ and the semigroup $S(X, \mathscr{F})$, the $S$-retracts of $X$ are precisely the closed subspaces of $X$.

This lemma and Theorem (4.4) combine to yield:

COROLLARY (4.9) Let $X$ be any Hausdorff space and let $f$ and $g$ be any two regular elements of $S(X, \mathscr{F})$. Then $f$ belongs to the two-sided ideal generated by $g$ if and only if Ran $g$ contains a closed subspace which is homeomorphic to Ranf. Two regular elements are $\mathscr{J}$-equivalent if and only if the range of each contains a closed subspace which is homeomorphic to the range of the other.

One easily checks that for any space $X$ whatsoever and the semigroup $S(X, \mathscr{P})$, the $S$-retracts are precisely the subsets of $X$. This and Theorem $(4,4)$ give:

COROLLARY (4.10) Let $X$ be any topological space and let $f$ and $g$ be any two regular elements of $S(X, \mathscr{P})$. Then $f$ belongs to the two-sided ideal generated by $g$ if and only if Ran $g$ contains a homeomorphic copy of $\operatorname{Ran} f$. The two functions are 
I-equivalent if and only if the range of each contains a homeomorphic copy of the ange of the other.

For any space $X$ and the semigroup $S(X, \mathscr{Z})$, a subset $A$ of $X$ is an $S$-retract if Ind only if $A$ is a retract of a clopen subspace of $X$. Furthermore, it follows from Corollary 2, p. 281, of Kuratowski (1966), that if $X$ is a 0-dimensional metric space, hen $A$ is an $S$-retract if and only if $A$ is a closed subspace of $X$. These observations together with Theorem $(4,4)$ yield:

Corollary (4.11) Let $X$ be a 0-dimensional metric space and let $f$ and $g$ be regular elements of $S(X, \mathscr{Z})$. Then $f$ belongs to the two-sided ideal generated by $g$ if and only if Rang contains a closed subspace which is homeomorphic to Ranf. The two functions are $\mathscr{J}$-equivalent if and only if the range of each contains a closed subspace which is homeomorphic to the range of the other.

We close this section with several results about the regular $\mathscr{D}$-classes of these various semigroups. We omit the result on $S(X)$ since it appears in Magill and Subbiah (1974) as Theorem (3.3). Nonetheless, this paper does intersect slightly with the latter paper with respect to a few of the other results on $S(X)$. The results we do state all follow in a straightforward manner from Theorem (4.5) and some observations made in this section so we dispense with their proofs.

COROLlaRY (4.12) Let $X$ be any topological space and let $A$ be any closed subspace of $X$. Let $D_{A}$ consist of all those functions in $S(X, \mathscr{F})$ such that $f$ maps some subset of $X$ homeomorphically onto $\operatorname{Ran} f$. Then $D_{A}$ is a regular $\mathscr{D}$-class of $S(X, \mathscr{F})$ and all regular $\mathscr{D}$-classes are obtained in exactly this manner.

COROLlaRY (4.13) Let $X$ be any topological space and let $A$ be any subspace of $X$. Let $D_{A}$ consist of all those functions $f$ in $S(X, \mathscr{P})$ such that $f$ maps some subset of $X$ homeomorphically onto $\operatorname{Ran} f$. Then $D_{A}$ is a regular $\mathscr{D}$-class of $S(X, \mathscr{P})$ and all regular $\mathscr{D}$-classes are obtained in exactly this manner.

Corollary (4.14) Let $X$ be a 0-dimensional metric space and let $A$ be any closed subspace of $X$. Let $D_{A}$ consist of all those functions $f$ in $S(X, \mathscr{Z})$ such that $f$ maps some subset of $X$ homeomorphically onto $\operatorname{Ran} f$. Then $D_{A}$ is a regular $\mathscr{D}$-class of $S(X, \mathscr{Z})$ and all regular $\mathscr{D}$-classes are obtained in exactly this manner.

\section{The maximal subgroups of sandwich semigroups of continuous functions}

The main result in this section is the following.

THEOREM (5.1) Let $S(X, Y, \alpha, \mathscr{E})$ be any sandwich semigroup of continuous functions and let $A$ be any subspace of $Y$ which $\alpha$ maps homeomorphically onto a 
retract of some $H$ in $\mathscr{E}$. Then the group $G(A)$ of all homeomorphisms mapping $A$ onto $A$ is isomorphic to a maximal subgroup of $S(X, Y, \alpha, \mathscr{E})$. Conversely, each maximal subgroup of $S(X, Y, \alpha, \mathscr{E})$ is isomorphic to some such $G(A)$.

Proof. By Theorem (4.1) of Magill and Subbiah (1975), the maximal subgroups of $S(X, Y, \alpha, \mathscr{E})$ are precisely the full $S$-isomorphism groups on $S$-retracts of $Y$. By Theorem (3.1) of this paper, a subspace $A$ of $Y$ is an $S$-retract if and only if $\alpha$ maps it homeomorphically onto a retract of some $H$ in $\mathscr{E}$. Hence, to complete the proof, we need only show that for such $A$, the group $J(A)$ of all $S$-isomorphisms mapping $A$ onto $A$ coincides with the group $G(A)$ of all homeomorphisms mapping $A$ onto $A$. Recall that an $S$-isomorphism from $A$ onto $A$ is the restriction of $f \circ \alpha$ for some $f$ in $S(X, Y, \alpha, \mathscr{E})$ where $f \circ \alpha$ maps $A S$-isomorphically onto $A$. By Theorem (3.4), the restriction of $f \circ \alpha$ to $A$ is a homeomorphism from $A$ onto $A$ so that $J(A)$ is contained in $G(A)$. Now let $t$ be any element of $G(A)$. Since $A$ is an $S$-retract, it is the range of some idempotent $v$ of $S(X, Y, \alpha, \mathscr{E})$ and it is immediate that both $t \circ v$ and $t^{-1} \circ v$ belong to $S(X, Y, \alpha, \mathscr{E})$. By Lemma (3.1) of Magill and Subbiah (1975), $v \circ \alpha$ is the identity on $A$ and so it readily follows that $(t \circ v) \circ \alpha \circ\left(t^{-1} \circ v\right) \circ \alpha$ is the identity on $A$ and $\left(t^{-1} \circ v\right) \circ \alpha \circ(t \circ v) \circ \alpha$ is also. This simply means that $(t \circ v) \circ \alpha$ maps $A S$-isomorphically onto itself and so that its restriction to $A$ belongs to the group $J(A)$. This restriction is, of course, just the homeomorphism $t$. Hence, $G(A)$ and $J(A)$ are one and the same and the proof is complete.

It follows as a corollary that the maximal subgroups of $S(X)$ are precisely the full homeomorphism groups on retracts of $X$. This fact appeared previously in Magill (1967) as Theorem (3.1), p. 138.

It has long been known that only symmetric groups can be maximal subgroups of full transformation semigroups. The situation differs, of course, for $S(X)$ in general and it is reasonable to wonder just what kinds of groups can be maximal subgroups of semigroups of continuous selfmaps. This question is answered by our next result.

THEOREm (5.2) Let $\mathscr{G}$ be any collection of groups. Then there exists a onedimensional, connected, locally connected, complete metric space $X$ such that each group in $\mathscr{G}$ is isomorphic to a maximal subgroup of the semigroup $S(X)$.

Proof. Index the groups in $\mathscr{G}$ by some index set $\Delta$. By Theorem 7, p. 96 of de Groot (1959), there exists for each $\delta \in \Delta$, a one-dimensional, connected, locally connected, complete metric space $Y_{\delta}$ such that the group $G\left(Y_{\delta}\right)$ of all homeomorphisms mapping $Y_{\delta}$ onto $Y_{\delta}$ is isomorphic to the group $G_{\delta}$ in $\mathscr{G}$. Let $X$ be any bonded union (Magill, 1974, Definition (3.1), p. 151) of the family $\left\{Y_{\delta}: \delta \in \Delta\right\}$. One verifies in a routine manner that since $Y_{\delta}$ is a one-dimensional, connected, locally connected, complete metric space, then $X$ is also. Moreover, one also shows easily 
that each $Y_{\delta}$ is homeomorphic to a retract of $X$ and this implies that $G\left(Y_{\delta}\right)$ and hence $G_{\delta}$ is isomorphic to the group of all homeomorphisms on a retract of $X$. We now apply either Theorem (3.1), p. 138 of Magill (1967) or Theorem (5.1) of this paper to conclude that each $G_{\delta}$ in $\mathscr{G}$ is isomorphic to a maximal subgroup of $S(X)$.

\section{Sandwich semigroups of Boolean ring homomorphisms}

By a Boolean ring, we mean any ring with identity with the property that every element is idempotent. Let $A$ and $B$ be any two Boolean rings and let $\theta$ be a homomorphism from $B$ into $A$. We denote by $Q(A, B, \theta)$ the sandwich semigroup of all homomorphisms from $A$ into $B$ where multiplication is defined by means of the sandwich homomorphism $\theta$. At first glance, it might seem that a discussion of these semigroups would be out of place in this paper but it is really not. Every sandwich semigroup can essentially be regarded as a sandwich semigroup of continuous functions. We use this fact and our previous results on sandwich semigroups of continuous functions to show that sandwich semigroups of Boolean ring homomorphisms are not often regular. This is contained in the main result of this section. Before we state it, some comments are in order. First of all, for any 0-dimensional Hausdorff space $X$, we denote by $\mathscr{B}(X)$ the Boolean ring of all clopen subsets of $X$ where, as usual, the sum of two elements is defined to be their symmetric difference and the product is defined to be their intersection. One other point, the adjective countable when used here means that the set under consideration is either finite or in one-to-one correspondence with the natural numbers. Countably infinite set are those which satisfy the second of the previous two conditions. We are now in a position to state the main result of this section.

THEOREM (6.1) Let $A$ and $B$ be two Boolean rings each with more than two elements and suppose that at least one of the rings is countable. Let $\theta$ be any homomorphism from the ring $B$ into the ring $A$. Then the following statements about the sandwich semigroup $Q(A, B, \theta)$ are equivalent.

(6.1.1) $Q(A, B, \theta)$ is regular.

(6.1.2) $\theta$ is an isomorphism from $B$ onto $A$ and either $A$ is finite or it contains exactly one maximal ideal $M$ with the property that each element in $M$ also belongs to a maximal ideal distinct from $M$.

(6.1.3) $\theta$ is an isomorphism from $B$ onto $A$ and $A$ is isomorphic to $\mathscr{B}(X)$ where $X$ is either finite or is the one-point compactification of the countably infinite discrete space.

We accomplish the proof through a sequence of lemmas. First of all, we need to recall the notion of the structure space of a commutative ring $R$ with identity. 
The points of the space which we denote by $X_{R}$ are the maximal ideals of $R$ and for each $r \in R$, the set $H_{r}=\left\{M \in X_{R}: r \in M\right\}$ is taken as a typical basic closed set. This space is always $T_{1}$ and compact (we do not demand here that a space be Hausdorff in order that it be compact) but it need not be Hausdorff. General information about structure spaces can be found in both Gillman and Jerison (1960) and Jacobson (1964). Of course, a Boolean ring is necessarily commutative and it is well known that the structure space $X_{A}$ of a Boolean ring $A$ is a compact 0-dimensional Hausdorff space.

It will be convenient to reserve the symbol $K_{r}$ for the set $X_{R}-H_{r}$. In other words, $K_{r}=\left\{M \in X_{R}: r \notin M\right\}$. It is well known that for any $a \in A, H_{a}=K_{b}$ where $b=1-a$. Consequently, each $H_{r}$ is clopen. In fact, the clopen subsets of $X_{A}$ are precisely the sets of the form $H_{r}$ (and hence also of the form $K_{r}$ ). Furthermore, it is also well known that for any Boolean ring $A$, the mapping $\rho$ defined by $\rho(a)=K_{a}$ is an isomorphism from $A$ onto $\mathscr{B}\left(X_{A}\right)$. The verification of the next result follows in a straightforward manner from this fact and will be omitted.

Lemma (6.2) Let $Q(A, B, \theta)$ be a sandwich semigroup of Boolean ring homomorphisms and define a mapping $\sigma$ from $\mathscr{B}\left(X_{B}\right)$ into $\mathscr{B}\left(X_{A}\right)$ by $\sigma\left(K_{b}\right)=K_{\theta(b)}$. Then $\sigma$ is a homomorphism from $\mathscr{B}\left(X_{B}\right)$ into $\mathscr{B}\left(X_{A}\right)$. Furthermore, the mapping $\Phi$ from $Q(A, B, \theta)$ into $Q\left(\mathscr{B}\left(X_{A}\right), \mathscr{B}\left(X_{B}\right), \sigma\right)$ which is defined by $\Phi(\tau)\left(K_{a}\right)=K_{\tau(a)}$ is an isomorphism from the former onto the latter.

LemMa (6.3) Let $X$ and $Y$ be compact 0-dimensional Hausdorff spaces. For each homomorphism $\varphi$ from $\mathscr{B}(X)$ into $\mathscr{B}(Y)$, there exists a unique continuous function $h$ mapping a clopen subset of $Y$ into $X$ such that $\varphi(H)=h^{-1}[H]$ for each $H \in \mathscr{B}(X)$. Furthermore, if $\varphi$ is an isomorphism from $\mathscr{B}(X)$ onto $\mathscr{B}(Y)$, then $h$ is a homeomorphism from $Y$ onto $X$.

The existence of the continuous function $h$ was essentially established in the proof of Lemma 1, p. 412, of Magill (1970b) and an analysis of that proof will easily convince one that if $\varphi$ is an isomorphism, then $h$ must be a homeomorphism. The uniqueness follows in a straightforward manner from the fact that the spaces are 0-dimensional and Hausdorff.

DefintTION (6.4) We will refer to the map $h$ in the previous lemma as the map which is induced by $\varphi$.

LeMMA (6.5) Let $\mathscr{Z}_{B}$ denote the collection of all clopen subsets of $X_{B}$ and let $t_{\sigma}$ denote the map from some clopen subset of $X_{A}$ into $X_{B}$ which is induced by the homomorphism $\sigma$ defined in Lemma (6.5). Then the mapping $\Gamma$ which sends an element of $Q\left(\mathscr{B}\left(X_{A}\right), \mathscr{B}\left(X_{B}\right), \sigma\right)$ into the function mapping $X_{B}$ into $X_{A}$ which it induces is 
an anti-isomorphism from $Q\left(\mathscr{B}\left(X_{A}\right), \mathscr{B}\left(X_{B}\right), \sigma\right)$ onto the sandwich semigroup $S\left(X_{B}, X_{A}, t_{\sigma}, \mathscr{Z}_{B}\right)$ which consists of all continuous functions whose domains are clopen subsets of $X_{B}$ and whose ranges belong to $X_{A}$.

Proof. Let $\alpha$ and $\beta$ be elements of $Q\left(\mathscr{B}\left(X_{A}\right), \mathscr{B}\left(X_{B}\right), \sigma\right)$ and denote $\Gamma(\alpha)$ and $\Gamma(\beta)$ by $f_{\alpha}$ and $f_{\beta}$ respectively. We want to show that $\Gamma(\alpha \beta)=\Gamma(\beta) \Gamma(\alpha)$. But this is equivalent to showing that

$$
\Gamma(\alpha \circ \sigma \circ \beta)=f_{\beta} \circ t_{\sigma} \circ f_{\alpha} .
$$

Let any $H \in \mathscr{B}\left(X_{A}\right)$ be given. Then by Lemma (6.3)

$$
(\alpha \beta)(H)=(\alpha \circ \sigma \circ \beta)(H)=\alpha(\sigma(\beta(H)))=f_{\alpha}^{-1}\left(t_{\sigma}^{-1}\left(f_{\beta}^{-1}(H)\right)\right)=\left(f_{\beta} \circ t_{\sigma} \circ f_{\alpha}\right)^{-1}(H) .
$$

Since the mapping induced by a homomorphism is unique, condition (6.8.1) follows and we see that $\Gamma$ is an anti-homomorphism. It is immediate that $\Gamma$ is injective and it also follows quite easily that $\Gamma$ is onto. Thus $\Gamma$ is an antiisomorphism.

Since any two anti-isomorphic semigroups must both be regular or both nonregular we immediately get

LeMma (6.6) $Q(A, B, \theta)$ is regular if and only if $S\left(X_{B}, X_{A}, t_{\sigma}, \mathscr{Z}_{B}\right)$ is regular.

LEMma (6.7) Let the Boolean ring $A$ have at least three elements. If $S\left(X_{B}, X_{A}, t_{\sigma}, \mathscr{Z}_{B}\right)$ is regular, then $t_{\sigma}$ is homeomorphism from $X_{A}$ onto $X_{B}$.

Proof. Suppose that $x \in X_{A}-\operatorname{Dom} t_{\sigma}$ and let $\langle x\rangle$ be the constant function which sends all points of $X_{B}$ onto $x$. Then $\langle x\rangle f\langle x\rangle=e$ for any $f$ in the semigroup so that $\langle x\rangle$ is not regular. Consequently the domain of $t_{\sigma}$ is all of $X_{A}$. Now suppose that $t_{\sigma}$ is not injective. Then $t_{\sigma}(x)=t_{\sigma}(y)$ for some $x, y \in X_{A}$ where $x \neq y$. But since $B$ has more than two elements, $X_{B}$ must have more than two elements and since $X_{B}$ is 0-dimensional it follows that $\{x, y\}$ is the range of a continuous function from $X_{B}$ into $X_{A}$. But this contradicts Theorems (3.1) and (3.5) and so we conclude that $t_{\sigma}$ is injective. Now suppose that $\operatorname{Ran} t_{\sigma}$ is not all of $X_{B}$. Since $X_{A}$ is compact and $X_{B}$ is 0-dimensional and Hausdorff, $\operatorname{Ran} t_{\sigma}$ is closed and there exists a nonempty clopen subset $H$ such that $H \subset X_{B}-\operatorname{Ran} t_{\sigma}$. Since $A$ has more than two elements, $X_{A}$ must have at least two. Denote them by $x$ and $y$ and let $f$ be the function which sends all points of $H$ into $x$ and those of $X-H$ into $y$. Then for any $g$ in the semigroup we have $x \notin \operatorname{Ran} f g f$ so that $f g f$ cannot possibly be $f$. Consequently, $\operatorname{Ran} t_{\sigma}=X_{B}$ and since $X_{A}$ is compact and $X_{B}$ is Hausdorff, we conclude that $t_{\sigma}$ is a homeomorphism from $X_{A}$ onto $X_{B}$.

LEMMA (6.8) $A$ Boolean ring $A$ is countably infinite and has exactly one maximal ideal $M$ with the property that each element in $M$ also belongs to a maximal ideal distinct from $M$ if and only if the structure space of $A$ is the one-point compactification of the countably infinite discrete space. 
Proof (Necessity). Let $M$ be such an ideal and let $M \in H_{\alpha}$. Then $a \in M$ and there exists a maximal ideal $N$ distinct from $M$ so that $a \in N$. This, of course means that $N \in H_{a}$ and since $\left\{H_{a}: a \in A\right\}$ is a basis for the open subsets, this means that $M$ is a limit point of $X_{A}$. However, there is only one such $M$ and this means that all other points of $X_{A}$ are isolated. Thus, since $X_{A}$ is compact, it must be the one-point compactification of some discrete space. But since $A$ is countable, $X_{A}$ must be metrizable. In fact, the structure space of a Boolean ring is metrizable if and only if the ring is countable (Sikorski, 1969, p. 25). Consequently, it follows from Theorem (8.6), p. 247 of Dugundji (1966) that $X_{A}$ is the one-point compactification of the countably infinite discrete space.

(Sufficiency). Now suppose that $X_{A}$ is the one-point compactification of the countably infinite discrete space. Then $X_{A}$ is metrizable and as have just observed, this means that $A$ is countable. Moreover, since $X_{A}$ has exactly one limit point $M$ and the sets of the form $H_{a}=\left\{N \in X_{A}: a \in N\right\}$ form a basis for the open sets of $X_{A}$, it readily follows that $M$ is the unique maximal ideal of $A$ such that each element of $M$ also belongs to a maximal ideal distinct from $M$.

Now we are finally in a position to complete the proof of Theorem (6.1). Suppose $Q(A, B, \theta)$ is regular. Then by Lemma (6.6), $S\left(X_{B}, X_{A}, t_{\sigma}, \mathscr{Z}_{B}\right)$ is also regular and by Lemma (6.7), $t_{\sigma}$ is a homeomorphism from $X_{A}$ onto $X_{B}$. It follows that $\sigma$ is an isomorphism from $\mathscr{B}\left(X_{B}\right)$ onto $\mathscr{B}\left(X_{A}\right)$ and this, in turn, implies that $\theta$ is an isomorphism from $B$ onto $A$. Since $t_{\sigma}$ is a homeomorphism, it also follows that $S\left(X_{B}, X_{A}, t_{\sigma}, \mathscr{Z}_{B}\right)$ is isomorphic to $S\left(X_{A}, \mathscr{Z}\right)$. In particular $f \rightarrow f_{\circ} t_{\sigma}$ is an isomorphism from the former onto the latter. Hence, $S\left(X_{A}, \mathscr{Z}\right)$ is regular and Theorem (3.11) tells us that $X_{A}$ is either discrete or is the one-point compactification of the countably infinite discrete space. But $X_{A}$ is compact under any circumstances so this means that $X_{A}$ is either finite or the one-point compactification of the countably infinite discrete space. Since $A$ is isomorphic to $\mathscr{B}\left(X_{A}\right)$, we have established that (6.1.1) implies (6.1.3).

Now we show that (6.1.3) implies (6.1.1). Since $\theta$ is an isomorphism from $B$ onto $A$, it easily follows that $Q(A, B, \theta)$ is isomorphic to the endomorphism semigroup of $A$ which we denote by $Q(A)$. Since $A$ is isomorphic to $\mathscr{B}(X), Q(A)$ is isomorphic to $Q(\mathscr{B}(X))$ but according to either Lemma $1, p .412$ of Magill (1970b) or Lemma (6.5) of this paper, the latter is isomorphic to $S(X, \mathscr{Z})$ which, in view of Theorem (3.12), is regular. Thus, $Q(A)$ is regular and this shows that (6.1.3) implies (6.1.1). It follows easily from Lemma (6.8) that (6.1.2) is equivalent to (6.1.3) and the proof of Theorem (6.1) is complete.

\section{Acknowledgement}

We wish to express our appreciation to the referee for his very detailed analysis of the original manuscript. His suggestions have resulted in what we feel is quite an improvement in the exposition of the results in this paper. 
References

G. Birkoff (1964), Lattice Theory (Amer. Math. Soc., Colloquium Publications 25, Ann Arbor, Mich.).

J. Dugundji (1966), Topology (Allyn and Bacon, Boston, Mass.).

L. Gillman and M. Jerison (1960), Rings of Continuous Functions (D. Van Nostrand, New York).

J. de Groot (1959), "Groups represented by homeomorphism groups, I", Math. Annal. 138, 80-102.

N. Jacobson (1964), Structure of Rings (Amer. Math. Soc., Colloquium Publications 37, Providence, R.I.).

K. Kuratowski (1966), Topology, 1 (Academic Press, New York).

K. D. Magill, Jr. (1967), "Semigroup structures for families of functions, II; continuous functions", J. Austral. Math. Soc. 7, 95-107.

K. D. Magill, Jr. (1970a), "Subgroups of semigroups of functions", Port. Math. 26, 133-147.

K. D. Magill, Jr. (1970b), "The semigroup of endomorphisms of a Boolean ring", J. Austral. Math. Soc. 11, 411-416.

K. D. Magill, Jr. (1974), "Semigroups which admit few embeddings", Fund. Math. 85, 37-53.

K. D. Magill, Jr. and S. Subbiah (1974), "Green's relations for regular elements of semigroups of endomorphisms", Canadian J. Math. 6 (26), 1484-1497.

K. D. Magill, Jr. and S. Subbiah (1975), Green's relations for regular elements of sandwich semigroups, I; general results", Proc. London Math. Soc. 3 (31), 194-210.

R. Sikorski (1969), Boolean Algebras (Springer-Verlag, New York).

State University of New York at Buffalo

Buffalo, New York 14214

USA

Daemen College

Buffalo, New York 14226

USA 